\title{
Research of Wireless Functions of Human Body Detection System Based on The Internet of Things
}

\author{
DUAN Jia-yong ${ }^{1, \text { a }}$, GUO Fang ${ }^{2, b}$ \\ ${ }^{1,2}$ Department of Electronics and Information Engineering, North China Institute of Science and \\ Technology, East Yanjiao, 101601, Beijing, China \\ ad1020702@ncist.edu.cn, bouofang1980@ncist.edu.cn
}

\section{Keywords: Human body function detection; Internet of things; ZigBee}

\begin{abstract}
A human body detection system based on internet of things is designed. System is divided into four parts: terminal controller, ZigBee network coordinator, ZigBee network control and display node and ZigBee sensor nodes. The system is mainly used CC2530 chip as information processing and exchange of units to achieve low-power wireless communications. Human performance testing system described in this article can be real-time monitoring of environmental parameters and body temperature, pulse and other information. Information is transmitted to the telematics terminal via ZigBee coordinator and end processor. Information can also be displayed directly on the display ZigBee network nodes.
\end{abstract}

\section{Introduction}

In recent years, internet of things becomes a hot area of global concern. Combination of internet of things technology and mine safety production activities can improve coal mine work environment. Coal miners can work in a better state, thereby reducing the risk factors, reduce the accident rate, and improve the safety leve ${ }^{[1-5]}$. From an ergonomic point of view, through the wireless body function detection system, you can mine electrical and mechanical equipment for a reasonable ergonomic design, and grasp the information and environmental information bodily functions coal miners. This will have important practical significance.

Specific configuration of wireless body function testing system is shown in Figure 1.

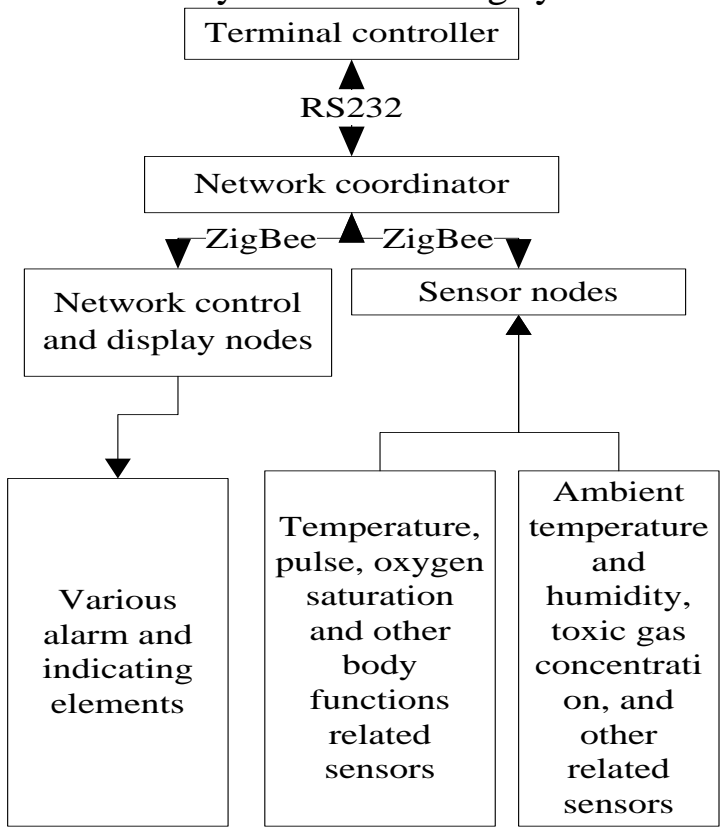

Figure1. Specific configuration of testing system

System consists of terminal controller, ZigBee network coordinator, ZigBee network control and display nodes and ZigBee sensor nodes and so on. ZigBee has a series of characteristics such as low power consumption, low complexity and high reliability ${ }^{[6-8]}$, it is very suitable for use in our system. 
The system can collect temperature, pulse, oxygen saturation, and other body functions related data, but also to collect temperature and humidity, toxic gas concentration, and other related information, and the information is transmitted in real time via the node to the terminal controller. On this basis, the information is transmitted to the processing center by the information processing center for data analysis, potential evaluation, identification and research.

\section{Hardware design}

The hardware part of the system consists of a terminal controller, coordinator and node boards, wireless network equipment and sensors. The system uses TI's CC2530 as node chips. CC2530-EB core board includes CC2530 microcontroller, wireless interfaces, crystal, and I / O expansion interface.

System related sensors can detect a variety of environmental and human performance related data, and send it to the coordinator via wireless means. The coordinator is the switch of information, and its function is to transfer the terminal controller to coordinate the command is sent to the control and wireless display terminal, but it also can transfer the implementation of the measured sensor signals and commands to the terminal controller.

\section{Software design}

Software flow chart is shown in Figure 2.

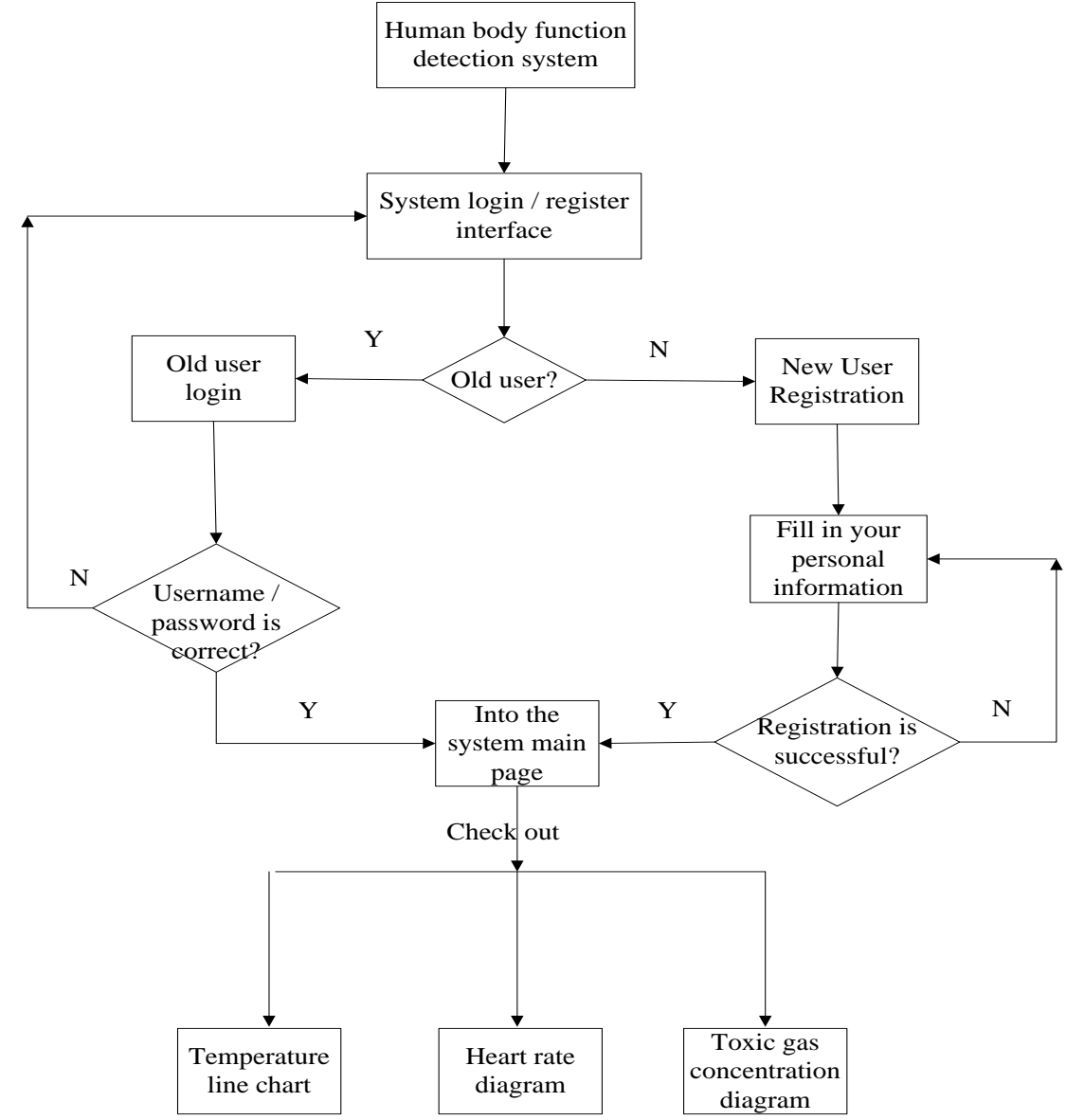

Figure2. Software flow chart of testing system

This software is designed to display the data as the core; using a line chart to plot the data collected is very conducive to observation. Software design also enables conventional system login, registration, and other functions to retrieve the password. Overall system functions relatively well. 
Software section complete with C \# language PC display systems, mainly the login screen, registration of personal information, and to view the body temperature, heart rate, blood oxygen content such as the main interface.

\section{System test}

Part of the system software testing: Win7, Visual studio, IAR Embedded Workbench. module.

The hardware system testing: ZigBee node, temperature sensor, heart rate measurement

In the temperature measurement, the temperature sensor larger, not like a thermometer placed in the human body as a direct measure axillary, using fingertip contact measurement, each measuring 30 minutes; in heart rate measurement, the fingers caught in the nail heart rate sensors measure, for 30 minutes for each measurement.

Body temperature data test results are shown in Figure 3.

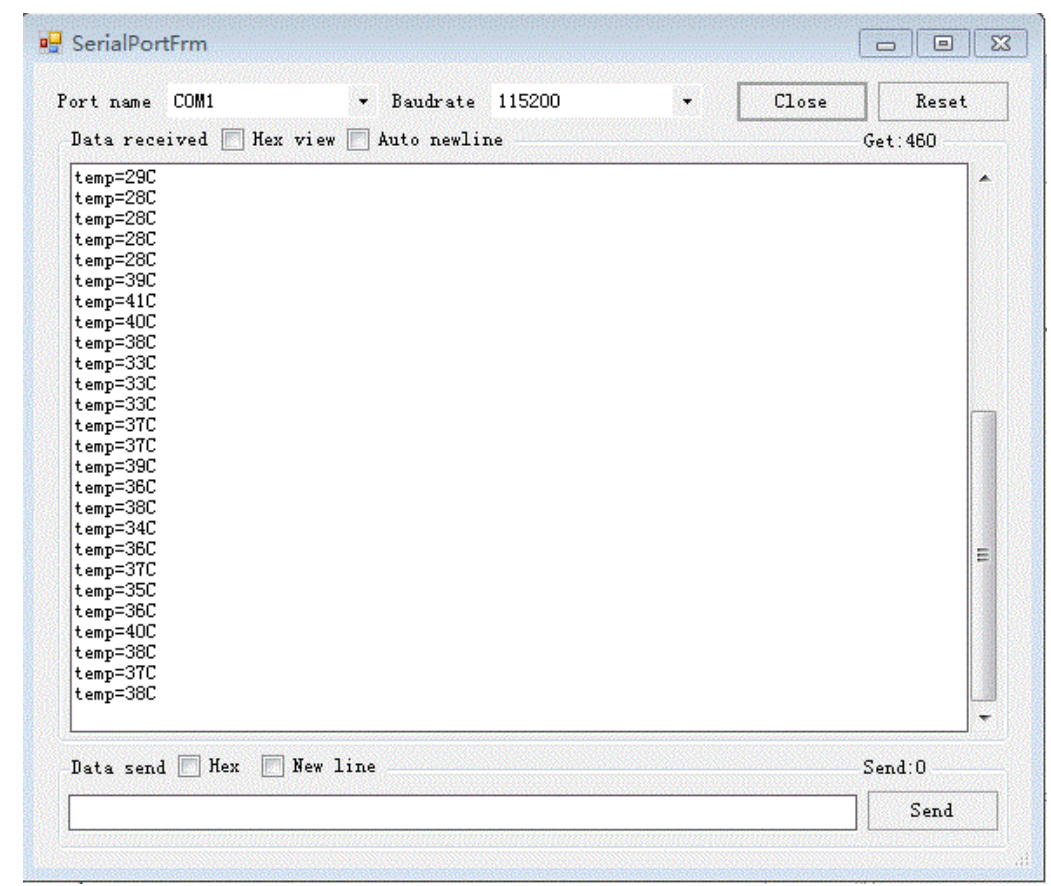

Figure3. Body temperature data test results

For temperature testing, we used to measure the finger temperature. As can be seen from the data obtained, the temperature of the transmission is very stable.

\section{Conclusion}

This article describes the wireless detection system based on human functioning of things. The system can collect data related to human performance, through Zigbee wireless network and spread to the PC serial port for display. This system uses a wireless transmission chip CC2530. The chip operates at $2.4 \mathrm{GHZ}$ band, with low power consumption and low cost. the software part based on Zigbee protocol stack can ensure the effectiveness and efficiency of the communication system. Follow-up study, the system can also increase the detection more physiological parameters, such as oxygen, pulse, electrocardiogram and electro-encephalogram.

\section{Acknowledgements}

In this paper, the research was sponsored by Fundamental research funds for the Central Universities (Project No. 3142014078, Project Name: The research of wireless human body detection system based on the Internet of things) 


\section{References}

[1] MA Xiaoping, HU Yan-jun, MIAO Yan-zi. Application research of technologies of Internet of Things, big data and cloud computing in coal mine safety production[J]. Industry and Mine Automation, 2014, 40(4): 5-9.

[2] ZHOU Li-xin,YANG Jun-qing. Sparing no effort in building "Internet of Things” of colliery safety production[J]. Jiangxi Coal Science \& Technology, 2010.4: 6-8.

[3]MENG Lei, DING En-jie, WU Li-xin. Research on key technologies of water inrush perception based on mine IoT[J]. Journal of China Coal Society, 2013,38(8):1397-1402.

[4]LI Jiang-hong. Research on Mine Gas Safety Monitoring Technology Based on Internet of Things[J]. Coal Technology, 2014.33(3): 212-214.

[5]LIU Yong-qiang. Research on the application of things in mine safety supervision[J]. Journal of Safety Science and Technology, 2012.09:9-14

[6]ZigBee Alliance. ZigBee Specifications (ZigBee Document 053474r17) [Z]. 2008-1-17

[7] Charlie Kaufman, Radia Perlman, Mike Speciner. Network Security Private Communication in a Public World, Second Edition[M]. Beijin: Publishing House of Electronices Industry, 2004:61-70.

[8] David Geer. Users Make a Beeline for ZigBee Technology[J]. Computer, 2005, 38(12): 16-19. 